\title{
Phototaxis in the Unicellular Red Algae Cyanidioschyzon merolae and Cyanidium caldarium
}

\author{
Mio Ohnuma ${ }^{1 *}$, Osami Misumi ${ }^{1,2}$ and Tsuneyoshi Kuroiwa ${ }^{1,3}$ \\ ${ }^{1}$ Research Information Center for Extremophile, Rikkyo University, \\ 3-34-1 Nishiikebukuro, Toshima-ku, Tokyo 171-8501, Japan \\ ${ }^{2}$ Graduate School of Medicine, Faculty of Science, \\ Department of Biological Science and Chemistry, Yamaguchi University, \\ 1677-1 Yoshida Yamaguchi, Yamaguchi 753-8512, Japan \\ ${ }^{3}$ JST, CREST, 7, Gobancho, Chiyoda-ku, Tokyo, 102-0076 Japan
}

Received March 2, 2011; accepted April 30, 2011

\begin{abstract}
Summary Phototaxis of 2 cyanidiaceae, Cyanidioschyzon merolae and Cyanidium caldarium, was studied by population experiments. We found that cells of both C. merolae and C. caldarium moved towards light in liquid medium, but that the degree of migration was quite different. When laterally illuminated, most of the $C$. merolae cells moved towards light at a velocity of $0.27 \mathrm{~mm} / \mathrm{h}$. In contrast, only a small proportion of $C$. caldarium cells showed migration towards light and most of the cells remained dispersed. The exterior cell surface of $C$. merolae was observed by scanning electron microscopy. It appeared thick and flexible enough to enable crawling movement.
\end{abstract}

Key words Cyanidioschyzon merolae, Cyanidium caldarium, Phototaxis, Red algae.

Phototaxis is a positive or negative movement along a light gradient. Although red algae are deficient in 3-dimensional phototaxis because they have no cilia or flagella (Jékely 2009), a few unicellular red algae, such as Porphyridium cruentum, display 2-dimensional phototaxis (Nultsch and Schuchart 1980). The mechanisms in red algae for sensing light, signaling and displacement are still unknown.

Cyanidioschyzon merolae and Cyanidium caldarium are unicellular extremophilic red algae, which inhabit acidic hot springs, and are considered to have diverged very early in the eukaryotic lineage (Nozaki et al. 2003). Both are very small (1-6 $\mu \mathrm{m}$ in diameter), and have a simple cell structure composed of 1 nucleus, 1 mitochondrion, 1 plastid and a minimum set of single-membrane-bound organelles: 1 microbody (peroxisome), 1 Golgi apparatus, a simple endoplasmic reticulum, and a small number of vacuoles (Kuroiwa et al. 1994). However, major differences between the 2 species are found in their cell forms, proliferation styles and cell surface. The club-shaped $C$. merolae cells proliferate by binary fission while the spherical $C$. caldarium cells manufacture endospores. C. merolae does not have a recognizable cell wall, but is coated with an electron-dense thin layer of filaments (Kuroiwa et al. 1994, Okuwaki et al. 1996), while C. caldarium has a cell wall approximately $50 \mathrm{~nm}$ thick. $P$. cruentum which is a phototactic red alga has a fibrillar sheath instead of cell wall surrounding the cell membrane (Gantt and Conti 1965). We were therefore interested to investigate whether $C$. merolae and $C$. caldarium with their differing cell surface structures showed phototaxis. We studied the phototaxis of $C$. merolae and $C$. caldarium by population experiments and also examined the cell surface of $C$. merolae by scanning electron microscopy.

*Corresponding author, e-mail: mioohnuma@rikkyo.ac.jp 


\section{Materials and methods}

Algal culture

C. merolae $10 \mathrm{D}$ and C. caldarium Delta were used in this study. C. merolae was originally collected in Italy (De Luca et al. 1978, Merola et al. 1981) and C. merolae 10D was isolated by Toda et al. (1995). C. caldarium Delta was freshly collected and isolated from the Owakudani valley in Hakone, Japan. All cells were routinely grown in MA2 medium (Ohnuma et al. 2008) in a glass vessel under continuous white light $\left(50 \mu \mathrm{mol}\right.$ photon $\left.\mathrm{m}^{-2} \mathrm{~s}^{-1}\right)$ at room temperature or at $40^{\circ} \mathrm{C}$.

Phototaxis experiments were carried out by leaving the cultures for a week at room temperature with lateral illumination without agitation. To confirm the phototaxic responses found in this initial experiment, cells of $C$. merolae and C. caldarium, which had been cultured with bubbling air, were diluted into L-tubes at $\mathrm{OD}_{750}=0.5$ and 0.6 , respectively (Fig. $2 \mathrm{~B}$ ). The tubes were placed vertically and incubated at $40^{\circ} \mathrm{C}$ without agitation in the dark overnight, so that the cells sank to the toe of the L-tube by gravity (Fig. 2A). The toe sections of the L-tubes were then wrapped with aluminum foil to exclude light from this area while the rest of the tube was illuminated, and the tube placed horizontally without agitation as shown in Fig. 2A.

\section{Electron microscopy}

To examine the exterior cell surface in detail, we disrupted C. merolae cells and observed them using scanning electron microscopy. Scanning electron microscopy was performed as previously described (Miyagishima et al. 1999). In brief, cells were cultured in $2 \times$ Allen's medium and synchronized (Suzuki et al. 1994). Collected cells were lysed in a French Pressure Cell (SLM) at

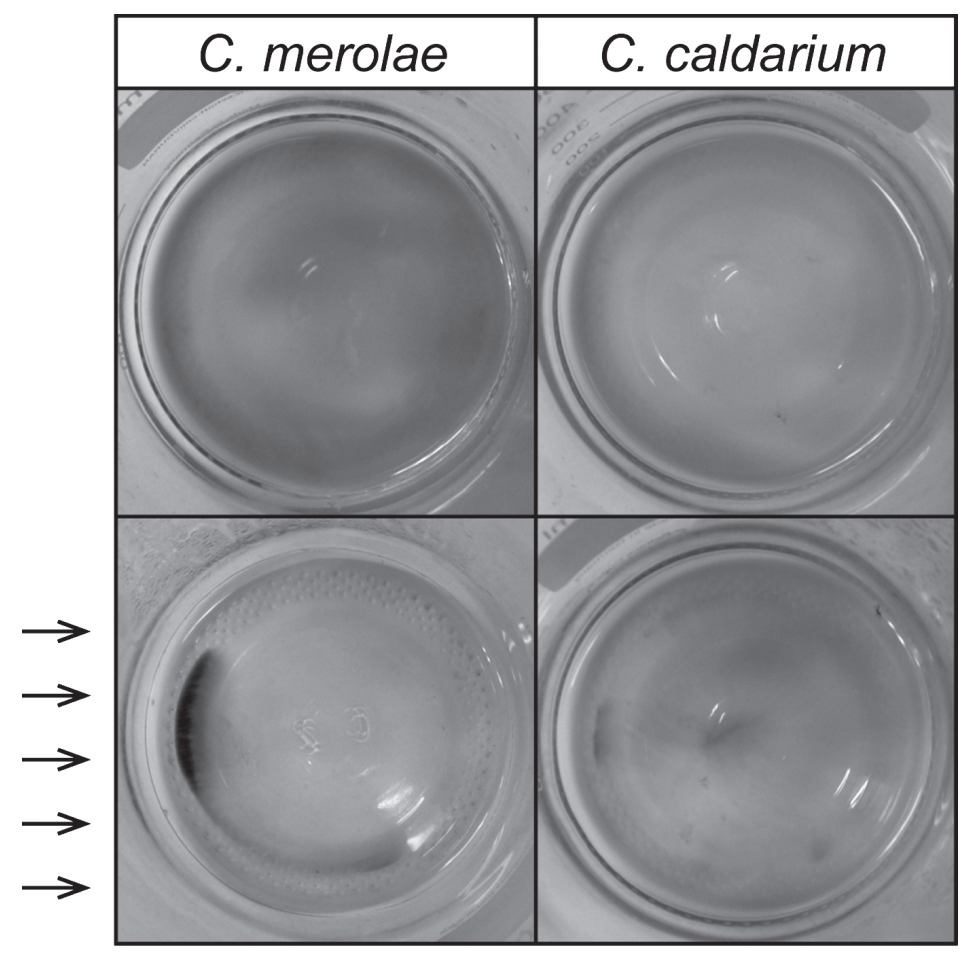

Fig. 1. Migration of C. merolae (left) and C. caldarium (right) in bottles. Cells which had been growing at $40^{\circ} \mathrm{C}$ with bubbling air under continuous illumination from above, were placed on one side of the incubator. Cultures were then illuminated from the lateral side of the bottles without agitation and left for a week. The photographs show top views of cultures taken on the first (top) and seventh (bottom) days. The direction of the light is indicated by arrows. 
A
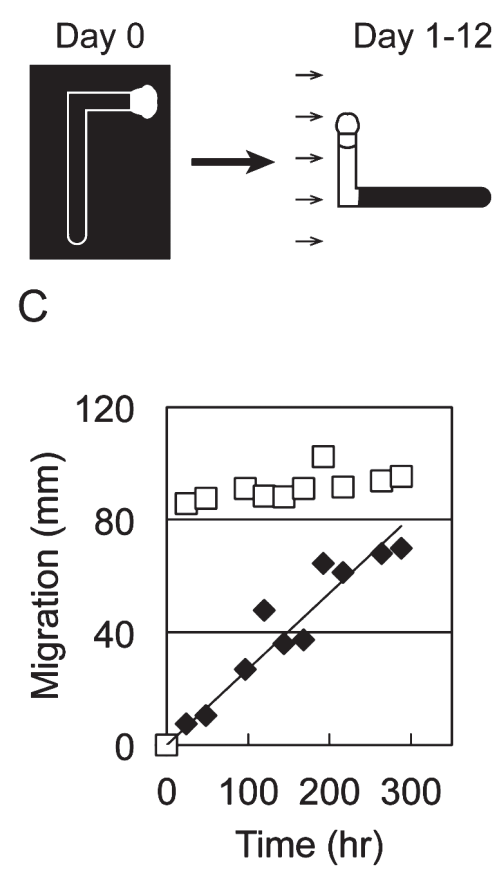

B

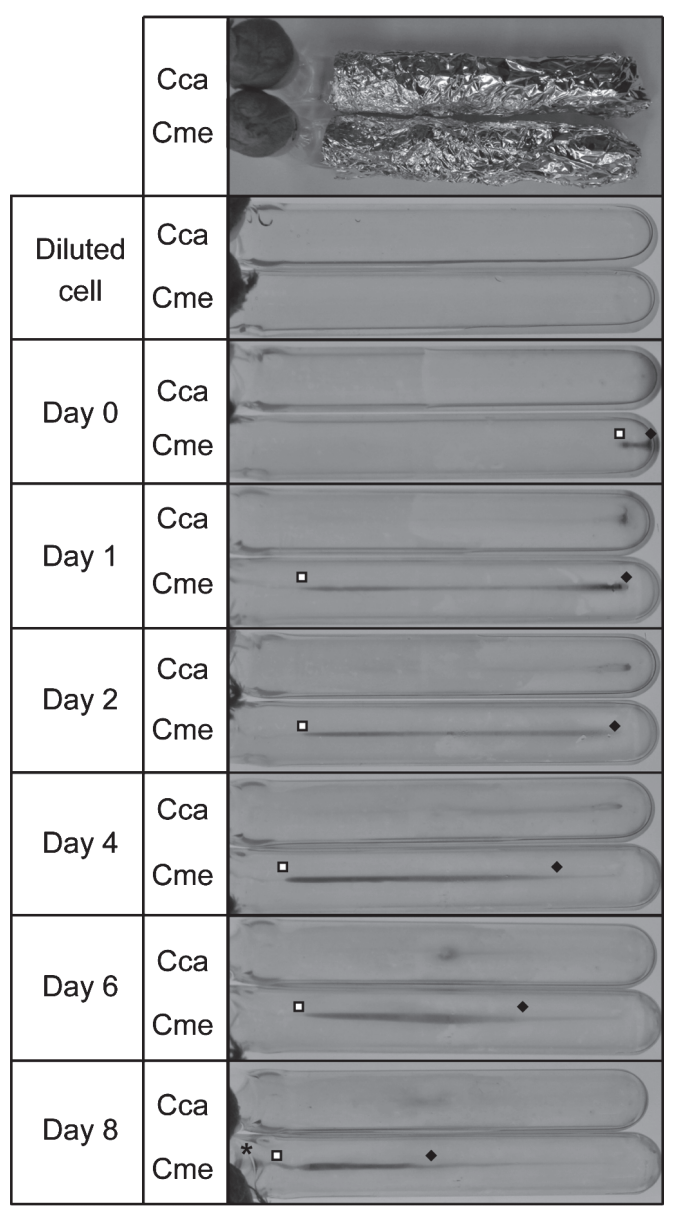

Fig. 2. Time course of phototaxis in C. merolae and C. caldarium. (A) Schematic representation of experiments to measure time course of phototaxis. Cells of $C$. merolae and C. caldarium in L-tubes diluted to $\mathrm{OD}_{750}=0.5$ and 0.6 , respectively, were placed vertically and incubated at $40^{\circ} \mathrm{C}$ overnight in the dark. Part of the L-tubes were wrapped with aluminum foil (indicated by black-colored area) before being placed horizontally and incubated for 12 days. The direction of the light is indicated by small arrows. (B) Representative views of L-tubes incubated as above. Positions of the leading edge (white square) and of the end (black diamond) of the cell mass are marked. Cells accumulated at the concave part of the L-tube (asterisk). (C) The time course of phototaxis in C. merolae. The position of the cells at the leading edge (white square), and at the end (black diamond) are indicated.

1500 psi (Miyagishima et al. 1999). Samples were collected by centrifugation and then fixed at room temperature for $2 \mathrm{~h}$. in $1 \%$ glutaraldehyde dissolved in $20 \mathrm{mM}$ sodium cacodylate $(\mathrm{pH} 7.2$ ). After fixation, the samples were washed 3 times with phosphate-buffered saline ( $\mathrm{pH}$ 7.2), and dehydrated in a graded ethanol series. Fixed, dehydrated cells were dried to the critical point, and then mounted and sputter-coated with platinum. The samples were examined with a JSM-6340F field emission scanning electron microscope (JEOL, Tokyo, Japan).

\section{Results and discussion}

Phototaxis of C. merolae and C. caldarium

When cultures of $C$. merolae and $C$. caldarium in bottles were left for a week at room tem- 

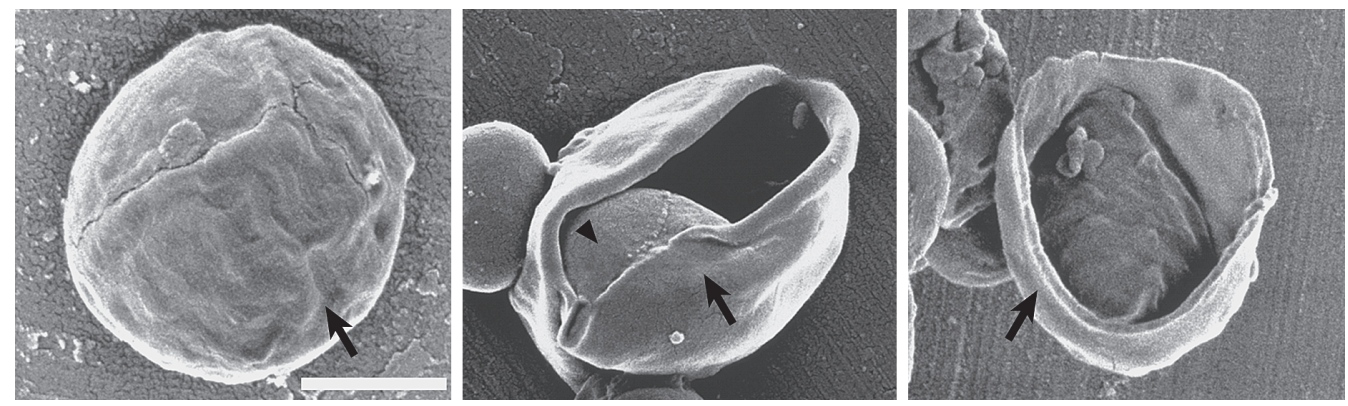

Fig. 3. Micrographs of C. merolae. Undisrupted cell (left), partly disrupted cell (middle) and completely disrupted cell (right) are shown. A plastid (arrowhead) is visible inside the cell surface structure (arrows). Bar $=0.5 \mu \mathrm{m}$.

perature with lateral illumination without agitation, we found that the majority of $C$. merolae cells migrated towards light, while most of the $C$. caldarium cells remained dispersed (Fig. 1). Although a small proportion of the $C$. caldarium cells migrated towards light, the effect was not nearly as dramatic as was observed in the $C$. merolae cells.

To confirm the phototaxis phenotypes of $C$. merolae and $C$. caldarium, cells of each species were placed in L-tubes as described in 'Materials and Methods' and left static overnight in the dark to locate the cells in the toe of the L-tubes (Fig. 2B Day 0). After exclusion of light from this area and horizontal placement of the tube in the light, cells were incubated for an additional $12 \mathrm{~d}$. On the first day (Fig. 2B Day 1), cells of both C. merolae and C. caldarium sank to the bottom of the L-tube and $C$. merolae cells started to migrate towards the curve of the tube. Individual cells of $C$. merolae varied in their degree of migration. Cells at the leading edge migrated near to the curve of the L-tube, although most of the cells remained at the toe. Cells of $C$. caldarium stayed near the toe of L-tube. From the second day to the eighth day (Fig. 2B Day 2-8), most of the C. merolae cells migrated over half the distance towards the curve of the L-tube. By the end of the experiment $C$. merolae cells had accumulated at the concave part of the L-tube (Fig. 2B Day 8, asterisk). By Day 8 , most of the $C$. caldarium cells had migrated less than half the length of the tube. Throughout the experiment, cells of $C$. caldarium remained dispersed. The result was that $C$. caldarium remained dispersed while $C$. merolae crawled bottom of the tube indicates that $C$. merolae showed stronger gravitaxis as well as phototaxis than $C$. caldarium. These observations were consistent with the results in the initial experiment (Fig. 1). We therefore used C. merolae for further analysis of the mechanism of phototaxis.

\section{Time course of phototaxis}

We measured the distance of migration of $C$. merolae to calculate its velocity. Since the degree of migration varied, distances were measured from the toe of the L-tube to both the position at the leading edge (Fig. 2B white squares) and the end (Fig. 2B black diamonds) of the cell mass. Time course-plots of $C$. merolae are shown in Fig. 2C. The time-course plot of cells at the leading edge reached a plateau on the first day (Fig. 2C white squares), when the fastest cells reached the curve of the L-tube. Migration of the slower cells of $C$. merolae showed good linearity (Fig. $2 \mathrm{C}$ black diamonds). Therefore, the plot of the end of the cell mass was used to calculate the velocity as the slope of the approximate expression. The velocity of $C$. merolae was calculated as $0.27 \mathrm{~mm} / \mathrm{h}\left(4.5 \mu \mathrm{m} / \mathrm{min}, R^{2}=0.91\right)$. The velocity of $C$. merolae was therefore 10 -fold greater than the $0.48 \mu \mathrm{m} / \mathrm{min}$ reported for P. cruentum (Nultsch and Schuchart 1980), which was measured on soft agar. 


\section{Cell surface of C. merolae}

Next, we examined the exterior cell surface of $C$. merolae. To examine the filamentous structure of the exterior cell surface of C. merolae (Okuwaki et al. 1996), the cells were disrupted in a French Press and viewed by scanning electron microscopy. In the French Press, most cells were disrupted, although a small number of cells remained undisrupted or partly disrupted. The cell surface of undisrupted cells appeared corrugated like the surface of a walnut (Fig. 3 left). When cells were partly disrupted (Fig. 3 middle), the cell surface structure was torn and the cell surface, which was corrugated before disruption, was smoothed out. Plastids of partly disrupted cells could be seen inside the torn cell surface structure. When cells were completely disrupted, plastids fell out of cells (Fig. 3 right). The cell surface of the completely disrupted cells seemed smooth as partly disrupted cells. The exterior cell surface structure appeared slightly larger than cell therefore the surface of intact cell wrinkles. When the cells were disrupted wrinkles of the cell surface were smoothed out from tear. The thickness of the cell surface structure was $40-80 \mathrm{~nm}$. These results were consistent with observations by transmission electron microscopy, in which the filaments of the exterior surface of C. merolae are approximately $100 \mathrm{~nm}$ in length and $8 \mathrm{~nm}$ in diameter (Okuwaki et al. 1996). The cell surface structure of $C$. merolae seemed to be thick enough and flexible enough to maintain regular shapes, such as club- and delta-shapes, which would enable crawling.

In most eukaryotic moving cells, actin filaments dynamically assemble and disassemble at the cell periphery to facilitate movement (Pollard and Borisy 2003). However, in spite of the existence of an actin gene in C. merolae (Takahashi et al. 1995), FITC conjugated phalloidin, which binds F-actin, did not react with the cell surface of C. merolae (Suzuki et al. 1995). It is possible therefore that $C$. merolae might use a non-conventional system for movement. Elucidating the molecular mechanism of phototaxis and gravitaxis in C. merolae using genomic information (Ohta et al. 1998, 2003, Matsuzaki et al. 2004, Nozaki et al. 2007) and genetic techniques (Minoda et al. 2004, Ohnuma et al. 2008, 2009, Yagisawa et al. 2009, Imamura et al. 2009, 2010, Yoshida, et al. 2009, 2010, Fujiwara et al. 2010) would inform future studies of the phototaxic and gravitaxtic mechanism in primitive algae.

\section{Acknowledgments}

We are grateful to Prof. Kawamura (Rikkyo University) for the availability of L-tubes. This work was supported by a Grant-in-Aid for Scientific Research (A) (22247007) awarded to T. K.

\section{References}

De Luca, P., Taddei, R. and Varano, L. 1978. Cyanidioschyzon merolae: A new alga of thermal acidic environments. Webbia 33: 37-44.

Fujiwara, T., Kuroiwa, H., Yagisawa, F., Ohnuma, M., Yoshida, Y,. Yoshida, M., Nishida, K., Misumi, O., Watanabe, S., Tanaka, K. and Kuroiwa, T. 2010. The coiled-coil protein VIG1 is essential for tethering vacuoles to mitochondria during vacuole inheritance of Cyanidioschyzon merolae. Plant Cell 22: 772-781.

Gantt, E. and Conti, SF. 1965. The ultrastructure of Porphyridium cruentum. J Cell Biol. 26: 365-381.

Imamura, S., Kanesaki, Y., Ohnuma, M., Inouye, T., Sekine, Y., Fujiwara, T., Kuroiwa, T. and Tanaka, K. 2009. R2R3type MYB transcription factor, CmMYB1, is a central nitrogen assimilation regulator in Cyanidioschyzon merolae. Proc. Natl. Acad. Sci. U.S.A. 106: 12548-12553.

—, Tarashita, M., Ohnuma, M., Maruyama, S., Minoda, A., Weber, A. P. M., Inouye, T., Sekine, Y., Fujita, Y., Omata, T. and Tanaka, K. 2010. Nitrate assimilatory genes and their transcriptional regulation in a unicellular red alga Cyanidioschyzon merolae: Genetic evidence for nitrite reduction by a sulfite reductase-like enzyme. Plant Cell Physiol. 51: 707-717.

Jékely, G. 2009. Evolution of phototaxis. Philos. Trans. R. Soc. Lond., B, Biol. Sci. 364: 2795-2808.

Kuroiwa, T., Kawazu, T., Takahashi, H., Suzuki, K., Ohta, N. and Kuroiwa, H. 1994. Comparison of ultrastructures between the ultrasmall eukaryote Cyanidioschyzon merolae and Cyanidium caldarium. Cytologia 59: 149-158.

Matsuzaki, M., Misumi, O., Shin-I, T., Maruyama, S., Takahara, M., Miyagishima, S. Y., Mori, T., Nishida, K., Yagisawa, 
F., Nishida, K., Yoshida, Y., Nishimura, Y., Nakao, S., Kobayashi, T., Momoyama, Y., Higashiyama, T., Minoda, A., Sano, M., Nomoto, H., Oishi, K., Hayashi, H., Ohta, F., Nishizaka, S., Haga, S., Miura, S., Morishita, T., Kabeya, Y., Terasawa, K., Suzuki, Y., Ishii, Y., Asakawa, S., Takano, H., Ohta, N., Kuroiwa, H., Tanaka, K., Shimizu, N., Sugano, S., Sato, N., Nozaki, H., Ogasawara, N., Kohara, Y. and Kuroiwa, T. 2004. Genome sequence of the ultra-small unicellular red alga Cyanidioschyzon merolae. Nature 428: 653-657.

Merola, A., Castaldo, R., De Luca, P., Gambardella, R., Musacchio, A. and Taddei, R. 1981. Revision of Cyanidium caldarium. Three species of acidophilic algae. G. Bot. Ital. (Florence, Italy) 115: 189-195.

Minoda, A., Sakagami, R., Yagisawa, F., Kuroiwa, T. and Tanaka, K. 2004. Improvement of culture conditions and evidence for nuclear transformation by homologous recombination in a red alga, Cyanidioschyzon merolae 10D. Plant Cell Physiol. 45: 667-671.

Miyagishima, S. Y., Itoh, R., Aita, S., Kuroiwa, H. and Kuroiwa, T. 1999. Isolation of dividing chloroplasts with intact plastid-dividing rings from a synchronous culture of the unicellular red alga Cyanidioschyzon merolae. Planta 209: 371-375.

Nozaki, H., Matsuzaki, M., Takahara, M., Misumi, O., Kuroiwa, H., Hasegawa, H., Shin-I, T., Kohara, Y., Ogasawara, N. and Kuroiwa, T. 2003. The phylogenetic position of red algae revealed by multiple nuclear genes from mitochondria-containing eukaryotes and an alternative hypothesis on the origin of plastids. J. Mol. Evol. 56: 485-497.

—, Takano, H., Misumi, O., Terasawa, K., Matsuzaki, M., Maruyama, S., Nishida, K., Yagisawa, F., Yoshida, Y., Fujiwara, T., Takio, S., Tamura, K., Chung, S. J., Nakamura, S., Kuroiwa, H., Tanaka, K., Sato, N. and Kuroiwa, T. 2007. A $100 \%$-complete sequence reveals unusually simple genomic features in the hot spring red alga Cyanidioschyzon merolae. BMC Biol. 5: 28.

Nultsch, W. and Schuchart, H. 1980. Photomovement of the Red Alga Porphyridium cruentum (Ag.) Naegeli II. Phototaxis. Arch. Microbiol. 125: 181-188.

Ohnuma, M., Misumi, O., Fujiwara, T., Watanabe, S., Tanaka, K. and Kuroiwa, T. 2009. Transient gene suppression in a red alga, Cyanidioschyzon merolae 10D. Protoplasma 236: 107-112.

-, Yokoyama, T., Inouye, T., Sekine, Y. and Tanaka, K. 2008. Polyethylene glycol (PEG)-mediated transient gene expression in a red alga, Cyanidioschyzon merolae 10D. Plant Cell Physiol. 49: 117-120.

Ohta, N. Matsuzaki, M., Misumi, O., Miyagishima, S. Y., Nozaki, H., Tanaka, K., Shin-I, T., Kohara, Y. and Kuroiwa, T. 2003. Complete sequence and analysis of the plastid genome of the unicellular red alga Cyanidioschyzon merolae. DNA Res. 10: 67-77.

- Sato, N. and Kuroiwa, T. 1998. Structure and organization of the mitochondrial genome of the unicellular red alga Cyanidioschyzon merolae deduced from the complete nucleotide sequence. Nucleic Acids Res. 26: 5190-5198.

Okuwaki, T., Takahashi, H., Itoh, R., Toda, K., Kawazu, T., Kuroiwa, H. and Kuroiwa, T. 1996. Ultrastructures of the Golgi body and cell surface in Cyanidioschyzon merolae. Cytologia 61: 69-74.

Pollard, T. D. and Borisy, G. G. 2003. Cellular motility driven by assembly and disassembly of actin filaments. Cell 112: $453-465$.

Suzuki, K., Ehara, T., Osafune, T., Kuroiwa, H., Kawano, S. and Kuroiwa, T. 1994. Behavior of mitochondria, chloroplasts and their nuclei during the mitotic cycle in the ultramicroalga Cyanidioschyzon merolae. Eur. J. Cell Biol. 63: 280-288.

—, Kawazu, T., Mita, T., Takahashi, H., Itoh, R., Toda, K. and Kuroiwa T. 1995. Cytokinesis by a contractile ring in the primitive red alga Cyanidium caldarium RK-1. Eur. J. Cell Biol. 67: 170-178.

Takahashi, H., Takano, H., Yokoyama, A., Hara, Y., Kawano, S., Toh-e, A. and Kuroiwa, T. 1995. Isolation, characterization and chromosomal mapping of an actin gene from the primitive red alga Cyanidioschyzon merolae. Curr. Genet. 28: 484-490.

Toda, K., Takahashi, H., Itoh, R. and Kuroiwa, T. 1995. DNA contents of cell nuclei in two Cyanidiophyceae: Cyanidioschyzon merolae and Cyanidium caldarium Forma A. Cytologia 60: 183-188.

Yagisawa, F., Nishida, K., Yoshida, M., Ohnuma, M., Shimada, T., Fujiwara, T., Yoshida, Y., Misumi, O., Kuroiwa, H. and Kuroiwa, T. 2009. Identification of novel proteins in isolated polyphosphate vacuoles in the primitive red alga Cyanidioschyzon merolae. Plant J. 60: 882-893.

Yoshida, Y., Kuroiwa, H., Hirooka, S., Fujiwara, T., Ohnuma, M., Yoshida, M., Misumi, O., Kawano, S. and Kuroiwa, T. 2009. A bacterial ZapA-like protein ZED is required for mitochondrial division. Curr. Biol. 19: 1491-1497.

—, Kuroiwa, H., Misumi, O., Yoshida, M., Ohnuma, M., Fujiwara, T., Yagisawa, F., Hirooka, S., Imoto, Y., Matsushita, K., Kawano, S. and Kuroiwa, T. 2010. Chloroplasts divide by contraction of a bundle of nanofilaments consisting of polyglucan. Science 329: 949-953. 